\title{
BIOPSIA RENAL PERCUTÂNEA MONITORIZADA POR ULTRASSONOGRAFIA EM CÃES
}

\author{
Alessandra Melchert, ${ }^{1}$ Flávio Quaresma Moutinho, ${ }^{2}$ Maria Jaqueline Mamprim ${ }^{2}$ E \\ Flávio Augusto Marques dos Santos ${ }^{3}$ \\ 1. UNOESTE.E-mail: alessandravet@unoeste.br \\ 2. UNESP Botucatu \\ 3. Universidade Anhembi Morumbi.
}

\section{RESUMO}

Com o objetivo de avaliar a técnica de biopsia renal (BR) percutânea monitorizada por ultrassonografia estudaram-se a qualidade das amostras obtidas e as complicações decorrentes do procedimento, em 43 cães adultos hígidos. Os animais foram divididos em dois grupos: Grupo I $(n=20)$ e II $(n=23)$, submetidos à biopsia do rim esquerdo e direito, respectivamente. Utilizou-se agulha do tipo "Tru-Cut", semiautomatizada e de 16 gauge. A amostra foi considerada de qualidade somente com a presença de pelo menos cinco glomérulos. Realizaram-se três tentativas de coleta de cada rim. Os resultados demonstraram que após a BR houve queda dos valores de hematócrito $(37,2 \pm 5,75$ para o Grupo 1 e $38,1 \pm 5,24$ para o Grupo 2, duas horas após a BR), sem consequências clínicas e ocorrência de hematúria e hematoma perirrenal transitórios, não mais observados em 72 horas. Do total de amostras de biopsia obtidas ( 81 fragmentos), $94 \%$ continham tecido renal e $32 \%$ (25 das 81 biopsias) e $44 \%$ das amostras ( 36 das 81 biopsias) dos Grupos 1 e 2 , respectivamente, apresentaram cinco ou mais glomérulos, perfazendo um total de $76 \%$ de material adequado para diagnóstico. Não foi observada a ocorrência de complicações maiores ou óbito, demonstrando a segurança da técnica. Concluiu-se que a técnica avaliada mostrou-se segura e de valor diagnóstico.

PALAVRAS-CHAVES: Biopsia renal, cães, percutânea, ultrassonografia.

\section{ABSTRACT}

\section{ULTRASOUND-GUIDED PERCUTANEOUS RENAL BIOPSY IN DOGS}

In order to evaluate the ultrasound-guided percutaneous renal biopsy technique in dogs, the complications of the procedure and the quality of the samples were studied in 43 health adult dogs, allocated into two groups: Group $1(n=20)$ and $2(n=23)$, that had three attempts of collection for the biopsy taken from the left end right kidney, respectively. The biopsies were obtained with "Trucut" semi-automatic, 16 gauge needles. The dogs were evaluated before and after the procedure and the quality of the sample was evaluated. The presence of at least five glomeruli was used as a criteria for sample quality. Following the renal biopsy, there was a decrease in the hematocrit, without clinical consequences, presence of blood in urine and transitory perirenal hematomas that resolved in 72 hours. There were no major complications or death, what demonstrates the safety of the technique. Ninety-four percent of the biopsy samples had renal tissue and $76 \%$ presented five or more glomeruli (32\% and $44 \%$ in Groups 1 and 2, respectively). In conclusion, the technique was safe and showed diagnostic value.

KEYWORDS: Dogs, percutaneous, renal biopsy, ultrasound. 


\section{INTRODUÇÃO}

As doenças renais são comuns em cães, sendo geralmente diagnosticadas por histórico, exame físico e testes laboratoriais (VADEN et al., 2005). Porém, muitas vezes os testes de rotina não são suficientes para o diagnóstico, fazendo-se necessária a avaliação morfológica dos rins por biopsia renal (BR) (GRAUER, 2001).

A BR é indicada quando os resultados possam alterar o tratamento ou facilitar o prognóstico, incluindo assim pacientes com glomerulopatias ou insuficiência renal aguda (IRA) (VADEN, 2004). A BR também avalia a resposta ao tratamento e verifica progressão (DiBARTOLA, 2004) e reversibilidade da doença renal (BARTGES \& OSBORNE, 1995).

A técnica de BR percutânea pode ser auxiliada pela ultrassonografia, o que resulta em menor índice de complicações hemorrágicas e melhor qualidade das amostras de BR, tanto no homem (MAYA et al., 2007) quanto nos animais, bem como detecta hemorragias após a coleta (OSBORNE et al., 1996).

Com ultrassom, a agulha de BR pode ser introduzida no órgão livremente (free-hand) ou com guia acoplado ao transdutor (BARR, 1995), que assegura o plano de incidência da agulha, mas atrapalha a manipulação. Os guias são específicos a determinadas probes e necessitam de software computadorizado para operação, o que aumenta os custos do procedimento (VADEN, 2004).

O uso de agulhas de 16 ou 18 gauge é preferido para a realização da técnica em animais. Com o uso de agulhas de 14 gauge há uma penetração mais profunda no parênquima renal, com maior probabilidade de penetração da agulha na medula renal, aumentando as chances de hemorragia (VADEN, 2004; VADEN et al., 2005).

A principal complicação da BR é a hemorragia (DiBARTOLA, 2004), devendo-se evitar o procedimento em animais com tendências hemorrágicas, anemia grave, coagulopatias (OSBORNE et al., 1996). VADEN (2004) contraindica a BR em pacientes com insuficiência renal crônica (IRC), nos quais há aumento dos riscos de complicações.

A amostra de BR é considerada de boa qualidade quando o número de glomérulos for suficiente para representar todo o parênquima renal (LAUFER-
AMORIM et al., 2002). Não existe um padrão estabelecido em medicina veterinária, sendo indicado em várias literaturas que o número mínimo de glomérulos por amostra deve ser de cinco (LAUFER-AMORIM et al., 2002; VADEN, 2004).

A literatura revela-se conflitante quanto ao calibre da agulha de BR e a qualidade das amostras. ZATELLI et al. (2005) relatam que, ao realizarem a BR com auxílio da ultrassonografia em cães, o uso de agulha do tipo Tru-Cut, semiautomatizada, de 18 gauge, proporcionou espécimes de biopsia adequados para diagnóstico. Entretanto, RAWLINGS et al. (2003), ao biopsiarem cães por laparoscopia ou com auxílio de ultrassonografia, relatam que os espécimes obtidos com agulha de mesmo calibre (18 gauge) apresentaram-se com poucos glomérulos e fragmentados, dificultando o diagnóstico.

O presente estudo teve como objetivos avaliar a técnica de BR percutânea monitorizada por ultrassom, as complicações decorrentes de sua aplicação e a qualidade das amostras obtidas, em cães hígidos.

\section{MATERIAL E MÉTODOS}

Foram utilizados 43 cães sem raça definida, hígidos, machos, adultos, peso corporal entre 8 e 20 $\mathrm{kg}$, provenientes do Canil do Biotério da Instituição de origem, divididos aleatoriamente em dois grupos - Grupo I ( $n=20)$ e Grupo II $(n=23)$-, nos quais foi realizada biopsia do rim esquerdo ou direito, respectivamente.

Depois de jejum, os animais foram anestesiados com cloridrato de xilazina, $1 \mathrm{mg} / \mathrm{kg}$, associado ao cloridrato de quetamina, $10 \mathrm{mg} / \mathrm{kg}$, por via intramuscular. Para a realização da BR, utilizou-se agulha de biopsia do tipo Tru-Cut $\left(\right.$ Baxter $\left.^{\circledR}\right)$, semiautomatizada, 16 gauge e 4,5 polegadas (Figura 1 ), com recolhimento semiautomático da cânula interna da agulha.

Os animais foram mantidos em decúbito dorsal, posicionando-se o transdutor ultrassonográfico na região mesogástrica, no sentido ventrodorsal (Figura 2A), isolando o rim de outros órgãos e facilitando a visualização da agulha de biopsia. A agulha foi inserida paralelamente à superfície do corpo do animal, no polo caudal do rim, mantendo trajeto paralelo ou perpendicular à pelve (Figura 2B), evitando-a. Realizaram-se duas a três punções por animal. 


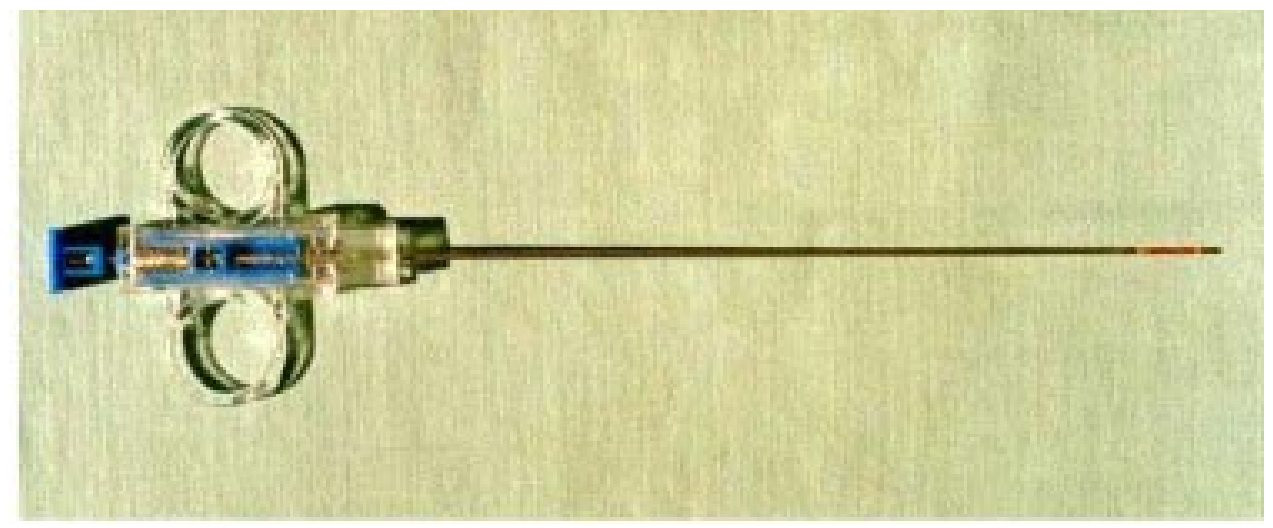

FIGURA 1. Agulha de biopsia do tipo “Tru Cut” semiautomatizada, após realização do procedimento, com fragmento de biopsia renal alojado na cânula interna exposta (ponta da seta).

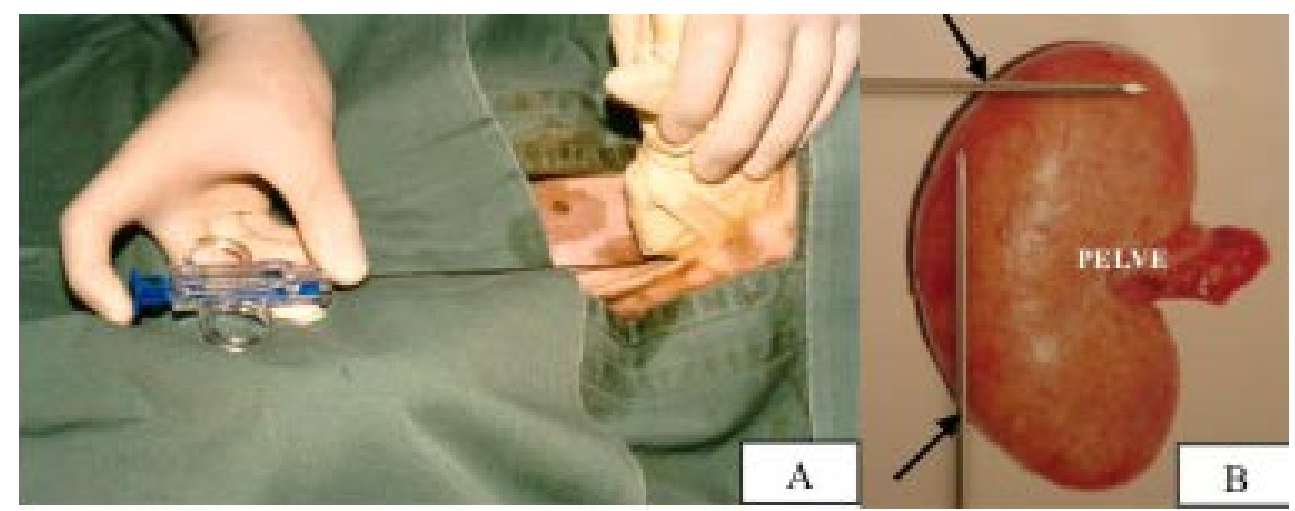

FIGURA 2. A) Posicionamento do trandutor do ultrassom e introdução da agulha de biopsia, no sentido cranial, no momento da coleta (animal posicionado em decúbito dorsal); B) Posicionamento adequado para inserção da agulha de biopsia no rim (setas), mostrando o trajeto que a agulha deve seguir no interior do parênquima renal, evitando-se a pelve.

Os animais foram avaliados antes da BR (M0 controle) e 2, 24, 72 e 144 horas após a realização da BR (M1, M2, M3 e M4, respectivamente). Avaliaramse o exame físico (frequência cardíaca; frequência respiratória; temperatura corporal; coloração das membranas mucosas), o hematócrito, a contagem global de leucócitos, a ureia e a creatinina séricas e urinálise. Procedeu-se à avaliação com o ultrassom da cavidade abdominal e dos rins por quinze minutos após a BR, para verificação de possíveis hemorragias.

As complicações observadas no estudo foram classificadas: em menores - hematomas perirrenais e hematúria macroscópica, que se resolveram espontaneamente, sem a necessidade de transfusão sanguínea; e maiores - hemorragias que requerem transfusão sanguínea (MOSTBECK et al., 1989; NYMAN et al., 1997).
O material obtido nas biopsias foi processado, submetido à coloração com hematoxilina-eosina e avaliados em microscopia de luz. Avaliou-se a qualidade das amostras pela presença ou ausência de tecido renal e pelo número de glomérulos presentes nas amostras positivas para rim. Consideraram-se, como de qualidade adequada para diagnóstico, cortes histológicos com pelo menos cinco glomérulos (LAUFER-AMORIM et al., 2002; VADEN, 2004).

Para as variáveis nas quais foram observadas as condições de normalidade utilizou-se a análise estatística multivariada de perfil (MORRISON, 1990), que permite comparação entre grupos e de cada grupo independente, nos diversos momentos de avaliação. Para as variáveis nas quais não se verificaram as condições de normalidade e/ou igualdade de variâncias, foi utilizada a análise estatística não paramétrica com aplicação do 
teste de Friedman, para comparação dos momentos de avaliação, e do teste de Mann-Withney, para comparação dos grupos em cada momento (ZAR, 1996). Considerou-se o nível de 5\% de significância.

\section{RESULTADOS}

Não foi observada a ocorrência de complicações maiores ou óbitos nos animais deste estudo. Dentre as complicações menores, observou-se a ocorrência de hematúria macroscópica transitória em oito $(18,6 \%)$ e hematoma perirrenal em um $(2,33 \%)$ dos 43 animais, perfazendo um total de $20,93 \%$.

Os cães estudados não apresentaram alterações do exame físico ou dos níveis de ureia e creatinina séricas. Na avaliação do leucograma, observou-se que os valores médios da contagem total de leucócitos permaneceram dentro dos limites da normalidade para cães em todos os momentos avaliados, não se verificando alterações que indicassem ocorrência de infecção após a BR.

Observou-se diminuição significativa do hematócrito nos animais do Grupo II $(\mathrm{p}<0,05)$ após a BR, em M1, e no Grupo I observou-se a mesma queda a partir de M1, porém sem diferença significativa. Nos demais momentos (M2, M3 e M4), registraram-se valores de hematócrito inferiores à média do momento controle, porém sem diferença significativa nos dois grupos.

Observaram-se um aumento significante no número de hemáceas na urina em M1, nos dois grupos, e diminuição gradativa nos momentos subsequentes, atingindo em M4 valores basais próximos ao normal. Todos os animais do estudo revelaram hematúria, sendo que a frequência de hematúria macroscópica foi de $18,6 \%$.

$\mathrm{Na}$ avaliação ultrassonográfica após a BR, nenhum dos animais mostrou hemoperitôneo e apenas um animal apresentou formação de hematoma subcapsular. Esse animal foi monitorado 24 horas após a biopsia, observando-se regressão significativa do hematoma e ausência de coágulos sanguíneos na bexiga urinária, não havendo a necessidade de transfusão sanguínea.

Não foram observadas diferenças na incidência de complicações ou na qualidade das amostras quando se compararam os dois grupos. De um total de 81 punções realizadas, obtiveram-se fragmentos renais em 76 espécimes (94\%). Dentre as 81 amostras, material ade- quado para diagnóstico (pelo menos cinco glomérulos por corte histológico) foi obtido em 32\% (25 das 81 biopsias) e $44 \%$ das amostras (36 das 81 biopsias) dos Grupos 1 e 2, respectivamente, perfazendo um total de $76 \%$, sendo que $6 \%$ apresentaram-se sem glomérulos, $12 \%$ apresentaram de um a quatro glomérulos e $6 \%$ das amostras apresentaram outros tecidos que não o renal (fígado, baço, gordura). A média de glomérulos para o grupo I foi de $11,88 \pm 9,64$ e para o grupo II, $15,74 \pm 9,29$.

\section{DISCUSSÃO}

A BR apresenta valor diagnóstico e prognóstico, além de fornecer orientações de auxílio à terapêutica. Entretanto, acredita-se que a BR não é frequentemente realizada em cães e gatos em virtude das complicações associadas ao procedimento e das dúvidas em relação ao diagnóstico (VADEN et al., 2005). Observou-se que, se utilizados materiais e técnica adequados, a BR não traz complicações graves e fornece material suficiente para diagnóstico.

A baixa incidência de complicações pode estar associada ao calibre das agulhas utilizadas (16 gauge), de acordo com HERGESELL et al. (1998), ou ainda ao uso de um sistema de agulha semiautomatizada, que torna a execução da biopsia mais rápida (BRADLEY \& O'HARA, 1996) e diminui a probabilidade de penetração da agulha na medula renal (WISEMAN et al., 1990), minimizando o traumatismo ao órgão e diminuindo a incidência das complicações. Também o uso da ultrassonografia nos procedimentos de BR percutânea proporciona menor incidência de complicações hemorrágicas (MAYA et al., 2007).

A hemorragia é a principal complicação após a realização da BR (SRINIVASAN et al., 1992; BARTGES \& OSBORNE, 1995). A hematúria foi a principal alteração observada. OSBORNE et al. (1996) citamna como uma complicação comum da BR em cães. Entretanto, a hematúria foi transitória e autolimitada, o que está de acordo com o descrito pela literatura (OSBORNE et al., 1974; OSBORNE et al., 1996; VADEN, 2004).

A hematúria microscópica ocorreu em todos os cães do estudo, após a BR. Este é um achado frequente nas primeiras 48 horas após a coleta. Já a hematúria macroscópica é menos comum e de peque- 
na significância em cães (OSBORNE et al., 1996). A hematúria microscópica autolimitada frequentemente ocorre no período de doze horas a três dias após a BR (OSBORNE et al., 1974; OSBORNE et al., 1996), o que também foi verificado neste estudo.

Mesmo submetidos a duas ou três punções por rim, apenas 18,6\% dos cães deste estudo revelaram a ocorrência de hematúria macroscópica. NASH et al. (1983) descreveram a ocorrência de hematúria macroscópica em $82,7 \%$ dos gatos submetidos a uma única punção renal. NASH et al. (1986), por sua vez, descreveram-na em 33,3\% dos gatos submetidos a várias punções. Isso demonstra que o número de punções não interfere com a intensidade da hemorragia após a biopsia.

Segundo OSBORNE et al. (1996), a magnitude da hemorragia pode ser monitorizada pela avaliação seriada do hematócrito de animais submetidos ao procedimento. A despeito da diminuição dos valores médios dessa variável, nenhum dos animais do estudo necessitou de transfusão sanguínea, o que classifica as hemorragias deste estudo como complicações menores, como postulado por MOSTBECK et al. (1989) e NYMAN et al. (1997).

Não foram observadas alterações nos valores do leucograma ou do exame físico que indicassem infecções após a BR. De modo similar, a literatura descreve baixa incidência de infecções após o procedimento em gatos (NASH et al., 1983; NASH et al., 1986) e no homem (GONZÁLEZ-MICHACA et al., 2000). Também a BR afeta minimamente a função renal (VADEN, 2004), o que foi observado neste estudo, uma vez que nenhum dos cães apresentou alterações dos valores de ureia e creatinina séricas. DROST et al. (2000) concluíram que a BR unilateral guiada por ultrassonografia tem efeito mínimo sobre a função renal de gatos hígidos, e GROMAN et al. (2004) relataram que biopsias seriadas de rins de cães jovens oferecem risco mínimo à indução de alterações renais.

A avaliação ultrassonográfica dos rins após a biopsia pode mostrar evidências de hemorragia, bem como sua gravidade (OSBORNE et al., 1996). A ocorrência de hematoma perirrenal em apenas um dos animais $(2,33 \%)$ está de acordo com os resultados obtidos por LÉVEILLÉ et al. (1993), que observaram a formação de hematomas perirrenais, imediatamente após o procedimento, em 5,6\% de cães e gatos com rins biopsiados. HOPPE et al. (1986) não observaram formação de hematomas como consequência de BR em cães, imediatamente ou 24 horas após o procedimento.

Deve-se considerar que pacientes com doença renal podem apresentar alterações como azotemia e hipertensão sistêmica, que aumentam os riscos de hemorragias. Essas alterações não contraindicam a realização do procedimento, porém exigem acirrada monitoração do paciente (VADEN, 2004). Apesar da relativa segurança da $\mathrm{BR}$, deve-se postergar sua realização na presença de trombocitopenia (BIGGE et al., 2001).

A técnica e os materiais utilizados para a biopsia renal neste estudo ofereceram resultados satisfatórios quanto à qualidade das amostras obtidas, revelando a presença de tecido renal em $94 \%$ das amostras e $76 \%$ de material adequado para diagnóstico (pelo menos cinco glomérulos por corte histológico). Trata-se de resultados semelhantes aos observados em cães e gatos na literatura (HOPPE et al., 1986; NASH et al., 1986; YAMAMOTO et al., 1991; MINKUS et al.,1994).

\section{CONCLUSÕES}

A BR per cutânea monitorizada por ultrassonografia, associada ao uso de agulhas semiautomatizadas, de 16 gauge, permitiu a obtenção de espécimes de biopsia adequados para o diagnóstico e evitou a ocorrência de complicações maiores ou óbitos, demonstrando a segurança da técnica e seu valor para diagnóstico.

\section{APOIO FINANCEIRO}

Fundação de Apoio à Pesquisa do Estado de São Paulo (Fapesp).

\section{REFERÊNCIAS}

BARR, F. Percutaneous biopsy of abdominal organs under ultrasound guidance. The Journal of Small Animal Practice, v. 36, n. 3, p. 105-113, 1995.

BARTGES, J. W.; OSBORNE, C. A. Canine and feline renal biopsy. In: OSBORNE, C. A.; FINCO, D. R. (Eds.). Canine and feline nephrology and urology. Baltmore: Lea \& Febiger, 1995. p. $277-302$. 
BIGGE, L. A.; BROWN, D. J.; PENNINCK, D. G. Correlation between coagulation profile findings and bleeding complications after ultrasound-guided biopsies: 434 cases (1993-1996). Journal of the American Animal Hospital Association, v. 37, n. 3, p. 228-233, 2001.

BRADLEY, W. A.; O'HARA, A. J. Comparison of four soft tissue core biopsy devices in canine liver and kidney biopsies. Australian Veterinary Practioner, v. 26, n. 1, p. 2-8, 1996.

DiBARTOLA, S. P. Abordagem clínica e avaliação laboratorial da doença renal. In: ETTINGER, S. J.; FELDMAN, E. C. (Eds.). Tratado de medicina interna veterinária. Rio de Janeiro: Guanabara Koogan, 2004. 2156 p.

DROST, W. T.; HENRY, G. A.; MEINKOTH, J. H. The effects of a unilateral ultrasound-guided renal biopsy on renal function in healthy sedated cats. Veterinary Radiology \& Ultrasound, v. 41, p. 57-62, 2000.

GONZÁLEZ-MICHACA, L.; CHEW-WONG, A.; SOLTERO, L.; GAMBA, G.; CORREA-ROTTER R. Percutaneous kidney biopsy, analysis of 26 years: complication rate and risk factors. Revista de Investigación Clínica, v. 52, n. 2, p. 125-31, 2000.

GRAUER, G. F. Testes diagnósticos para o sistema urinário. In: NELSON, R. W.; COUTO, C. G. (Ed.). Medicina interna de pequenos animais. 2. ed. Rio de Janeiro: Guanabara Koogan, 2001. p. 466-479.

GROMAN, R. P.; BAHR, A.; BERRIDGE, B. R.; LEES, G. E. Effects of serial ultrasound-guided renal biopsies on kidneys of healthy adolescent dogs. Veterinary Radiology \& Ultrasound, v. 45, n. 1, p. 62-69, 2004.

HERGESELL, O.; FELTEN, H.; ANDRASSY, K.; KÜHN, K.; RITZ, E. Safety of ultrassound-guided percutaneous renal biopsyretrospective analysis of 1090 consecutive cases. Nephrology Dialysis Transplantation, v. 13, n. 4, p. 975-977, 1998.

HOPPE, F. E.; HAGER, D. A.; POULOS, P. W.; EKMAN, S.; LINDGREN, P. G. A. Comparisation of manual and automatic ultrasound-guided biopsy techniques. Veterinary Radiology, v. 27, n. 4, p. 99-101, 1986.

LAUFER-AMORIM, R.; BANDARRA, E. P.; MONTEIRO JÚNIOR, L. A.; DE MOURA, V. M. B. D. Avaliação quantitativa e qualitativa da técnica de biopsia renal "de janela" em cães. Ciência Animal Brasileira, v. 3, n. 2, p. 41-45, ????.

LÉVEILlÉ, R.; PARTINGTON, B. P.; BILlER, D. S.; MIYABAYASHI, T. Complications after ultrasound-guided biosy of abdominal strutures in dogs and cats: 246 cases (1984-1991). Journal of the American Veterinary Medical Association, v. 203, n. 3, p. 413-415, 1993.
MAYA, I. D.; MADDELA, P.; BARKER, J.; ALLON, M. Percutaneous renal biopsy: comparison of blind and real-time ultrasound-guided technique. Seminars in Dialysis, v. 20, n. 4, p. 355-358, 2007.

MINKUS, G.; REUSCH, C.; HÖRAUF, A.; BREUER, W.; DARBÈS, J.; KRAFT, W.; HERMANNS, W. Evaluation of renal biopsies in cats and dogs - histopathology in comparison with clinical data. The Journal of Small Animal Practice, v. 35, p. 465-472, 1994.

MORRISON, D. F. Multivariate statistical methods. São Paulo: Mc Graw-Hill, 1990.

MOSTBECK, G. H.; WITTICH, G. R.; DERFLER, K.; ULRICH, W.; WALTER, R. M.; HEROLD, C.; HALLER, J.; TSCHOLAKOFF, D. Optimal needle size for renal biopsy: in vitro and in vivo evaluation. Radiology, v. 173, n. 3, p. 819-22, 1989.

NASH, A. S.; BOYD, J. S.; MINTO, A. W.; WRIGHT, N. G. Renal biopsy in the normal cat: an examination of the effects of a single needle biopsy. Research in Veterinary Science, v. 34, p. 347-356, 1983.

NASH, A. S.; BOYD, J. S.; MINTO, A. W.; WRIGHT, N. G. Renal biopsy in the normal cat: examination of the effects of repeated needle biopsy. Research in Veterinary Science, v. 40, n. 1, p. 112-117, 1986.

NYMAN, R. S.; CAPPELEN-SMITH, J.; AL SUHAIBANI, H.; ALFURAYH, O.; SHAKWEER, W.; AKHTAR, M. Yield and complictions in percutaneous renal biopsy. A comparison between ultrasound-guided gun-biopsy and manual tchniques in native and transplant kidneys. Acta Radiologica, v. 38, p. 431-436, 1997.

OSBORNE, C. A.; STEVENS, J. B.; PERMAN, V. Kidney Biopsy. The Veterinary Clinics of North America: Small Animal Practice, v. 4, n. 2, p. 351-65, 1974.

OSBORNE, C. A.; BARTGES, J. W.; POLZIN, D. J.; LULICH, J. P.; JOHNSTON, G. R.; COX, V. Percutaneous needle biopsy of the kidney. The Veterinary Clinics of North America: Small Animal Practice, v. 26, n. 6, p. 1461-1504, 1996.

RAWLINGS, C.; DIAMOND, H.; HOWERTH, E.; NEUWIRTH, L.; CANALIS, C. Diagnostic quality of percutaneous kidney biopsy specimens obtained with laparoscopy versus ultrasound guidance in dogs. Journal of the American Veterinary Medical Association, v. 223, n. 3, p. 317-321, 2003.

SRINIVASAN, S. R.; RAJAN, T. S. S.; DHANAPLAN, P.; GNANAPRAKASAN, V. Evaluation of different renal biopsy techniques in canine. The Indian Veterinary Journal, v. 69, p. 449-451, 1992. 
VADEN, S. L. Renal biopsy: methods and interpretation. The Veterinary Clinics of North America: Small Animal Practice, v. 34, n. 4, p. 887-980, 2004.

VADEN, S. L.; LEVINE, J. F.; LEES, G. E.; GROMAN, P. R.; GRAUER, G. F.; FORRESTER, S. D. Renal biopsy: a retrospective study of methods and complications in 283 dogs and 65 cats. Journal of Veterinary Internal Medicine, v. 19, n. 6, p. 794-781, 2005

WISEMAN, D. A.; HAWKINGS, R.; NUMEROW, L. M.; TAUB, K. J. Percutaneous renal biopsy utilizing real time, ultrasonic guidance and a semi automated biopsy device. Kidney International, v. 38, p. $347-349,1990$.
YAMAMOTO, K.; ISHIYAMA, N.; YAMAGA, Y.; HAYASHI, T.; KAGOTA, K. Ultrasound-guided techniques for biopsy of the kidney of the medium-sized dog. The Journal of Veterinary Medical Science, v. 53, n. 2, p. 345-346, 1991.

ZAR, J. H. Biostatistical analysis. New Jersey: Prentice-Hall, 1996.

ZATELLI, A.; D 'IPPOLITO, P.; ZINI, E. Comparison of glomerular number and specimen lenght obtained from 100 dogs via echo-assisted renal biopsy using two different needles. Veterinary Radiology \& Ultrasound, v. 46, n. 5, p. 434-436, 2005. 